\title{
Performance Evaluation of MGfast Systems Over Coaxial Cables
}

\author{
Marx Freitas, Aline Ohashi, Diogo Acatauassu, João C. W. A. Costa, Eduardo Medeiros, Miguel Berg, \\ Igor Almeida and André M. Cavalcante
}

\begin{abstract}
A trend for the 5th generation fixed broadband access networks is to provide data rates as high as 10 Gbps to the subscribers. Since fiber-to-the-home (FTTH) connectivity is still expensive, the use of alternative architectures is attractive in some scenarios. In this context, the International Telecommunication Union (ITU) is standardizing the MGfast access technology to provide multi-gigabit transmission over copper media. This paper evaluates the performance of MGfast systems adopting RG-59, RG-6 and RG-11 coaxial cables as interface between the distribution point units (DPUs) and the customer premises equipment (CPE). The operation mode of MGfast is assumed to be synchronized time division duplexing (STDD), the system is based on discrete multi-tone (DMT) and a simple loading method for power allocation is employed. Simulation results show that, for loop lengths less than $100 \mathrm{~m}$, data rates as high as 11 Gbps can be achieved using a transmission profile that goes up to $848 \mathrm{MHz}$ and gives support to 16384 quadrature amplitude modulation (QAM) per DMT sub-channel. Assuming a profile that goes up to $1696 \mathrm{MHz}$ and gives support the aforementioned modulation scheme, the data rates can be as high as 23 Gbps.
\end{abstract}

Index Terms-MGfast, Coaxial Cables, Fixed Broadband, Hybrid Fiber-Coaxial Networks.

\section{INTRODUCTION}

$\mathbf{O}$ VER the last years, the continuous demand for data traffic has motivated enhancements on fixed broadband access networks originally projected for plain old telephone service (POTS) and television (TV) services. According to [1]-[4], the fifth generation fixed broadband is designed to increase the aggregate data rates towards tens of Gbps. In this scenario, the International Telecommunication Union (ITU) is standardizing the MGfast broadband access technology, within the G.mgfast project, to enable costly-effective multi-gigabit transmission on existing copper cables [3]-[11].

The MGfast aims at providing data rates as high as $10 \mathrm{Gbps}$ using architectures such as fiber-to-the-building (FTTB) and fiber-to-the-distribution point (FTTdp), as well as extending the G.fast frequency limit from $212 \mathrm{MHz}$ up to $424 \mathrm{MHz}, 848$ $\mathrm{MHz}$ and (possibly) $1696 \mathrm{MHz}$ [3], [4], [11]-[13]. In addition

M. Freitas, A. Ohashi, D. Acatauassu and J. C. W. A. Costa are with the Applied Electromagnetism Laboratory, Federal University of Pará, Brazil (email: \{marx; alineohashi; diogoaca; jweyl\}@ufpa.br).

E. Medeiros and M. Berg are with the Ericsson Research, Ericsson AB, Sweden (e-mail: \{eduardo.medeiros; miguel.berg\} @ericsson.com).

I. Almeida and A. M. Cavalcante are with the Ericsson Research, Ericsson Telecomunicações S.A., Brazil (e-mail: \{igor.almeida; andre.mendes.cavalcante\} @ ericsson.com).

This work was supported by the Innovation Center, Ericsson Telecomunicações S.A., Brazil, by the Council for Scientific and Technological Development $(\mathrm{CNPq})$ and by the Coordination of Superior Level Staff Improvement (CAPES).

Digital Object Identifier: 10.14209/jcis.2021.5 to this, MGfast aims at operating under low power consumption, leading distribution point units (DPUs) to be supplied by the users through the copper cables, using technologies such as reverse power feeding (RPF) [3], [4], [11].

Recent works showed promising results, indicating by simulation that full-duplex MGfast systems operating over coaxial cable networks can effectively achieve data rates in the range of tens of Gbps [3]-[5]. However, to the best of our knowledge, simulations exhibiting the associated transmit powers are still scarce. In order to cover this gap, this paper evaluates achievable data rates and transmit powers of MGfast access systems employing RG-59, RG-6 and RG-11 coaxial cables as copper media. The analyses consider transmission profiles that goes up to $424 \mathrm{MHz}, 848 \mathrm{MHz}$ and $1696 \mathrm{MHz}$, cover a variety of loop lengths, adopt spectral constraints and explore distinct configurations for the cyclic prefix. Furthermore, in order to consider a less sophisticated implementation, the operation mode of MGfast is assumed to be synchronized time division duplexing (STDD).

The contributions of this paper are: $i$ ) show the potential of MGfast systems over RG-59, RG-6 and RG-11 coaxial cables through computer-based simulations, $i i$ ) use a loading method to minimize the transmit power over the lines, iii) show that for short distances, the cable type does not affect the system's transmission capacity, $i v$ ) analyze how the maximum number of bits per discrete multi-tone modulation (DMT) sub-channel, cyclic prefix length and cable type impact on the aggregate net data rate and transmit power.

The remaining of this paper is organized as follows. Section II reviews the network architecture adopted by MGfast. It describes the coaxial cable types considered to be used in real deployments and exposes the parametric cable model employed here for simulating the signal loss characteristics of such lines. Section III exhibits the MGfast system model used in this work and presents the simulation parameters defined in the tests. Such parameters include the transmission profiles, the power spectrum densities (PSDs), the cable lengths and the loading method adopted for power allocation. Continuing, Section IV presents the performance evaluation results in terms of the achievable data rates and transmit powers. It shows the benefits of using the loading method described in Section III in order to obtain a power-efficient implementation. At last, Section V concludes the paper. 


\section{MGfast Over Coaxial Cables}

\section{A. Network Architecture}

The network architectures considered to be used in MGfast are similar to those ones used in G.fast [12], where optical fiber cables flow from a central office (CO) toward a DPU, as shown in Fig. 1. The DPU establishes the connection between the customer premises equipment (CPE) and the rest of the access network through a copper media (e.g., coax) and it must be placed near to the customer premises. Hence, the DPUs are usually installed in locations such as external walls, in basements and in the building floor [14].
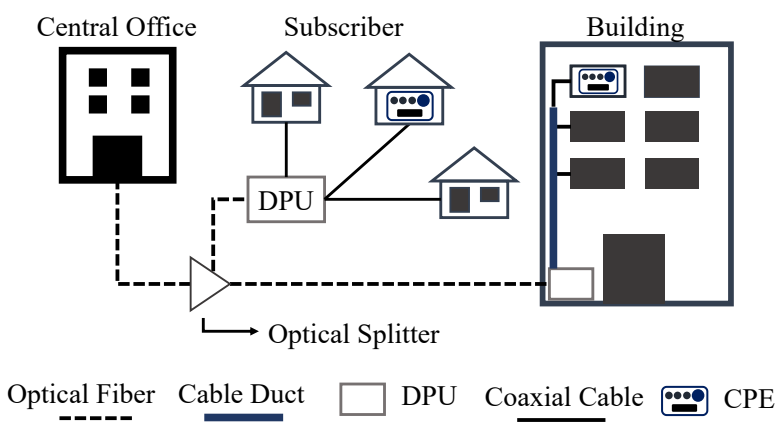

Fig. 1. Network architecture adopted for MGfast systems over coax.

In MGfast, the copper loops employed between the DPUs and the CPE are designed to range from $30 \mathrm{~m}$ to $100 \mathrm{~m}^{1}$ and the deployments may support both single-dwelling unit (SDU) and multi-dwelling unit (MDU) [11], [14]. Moreover, MGfast is projected to coexist with other transmission systems such as G.fast or satellite TV (SAT TV) [4], [15].

\section{B. Cable Types and Line Modeling}

In order to establish MGfast operations over coaxial networks, the ITU suggests the RG-6, RG-59 and RG-11 coaxial cable types. These lines are widely employed nowadays in cable TV, SAT TV, baseband video in closed-circuit TV and cable modems [11]. The RG-11 has the best performance and, in general, the highest cost, whereas the RG-59 is the one with the highest attenuation among the above mentioned.

In this work, the insertion loss $(I L)$ characteristics of such lines were estimated by means of the KM1 cable model [16][18], as follows:

$$
I L(f)=k_{1} \sqrt{f}+k_{2} f \quad(\mathrm{~dB} / 100 \mathrm{~m}),
$$

where $f$ is the frequency in $\mathrm{MHz}$, and $k_{1}$ and $k_{2}$ are the model parameters related to the skin effect attenuation and dielectric loss attenuation, respectively.

We choose to use the KM1 cable model instead of the exponential approximation model suggested by the ITU, given by:

$$
I L(f)=a \times f^{b}+c \times f+d \quad(\mathrm{~dB} / 100 \mathrm{~m}),
$$

\footnotetext{
${ }^{1}$ Operation over metallic links up to at least $400 \mathrm{~m}$ may be possible, subject to some performance restrictions [11].
}

where $a, b, c$ and $d$ are coefficients (fitting parameters) related to each cable type and $f$ is the frequency in $\mathrm{MHz}$, since the fitted parameters values presented in [15] are somehow atypical and non-physical. For example, the value of $b$ should be 0.5 for all cables since $a$ and $b$ model the skin effect, while $c$ models the dielectric loss (related to the shunt conductance). Probably, this inconsistency was propagated from the table showing attenuation vs frequency numbers presented in [15]. It is possible to note that the RG-6 attenuation values at $900 \mathrm{MHz}$ and $1 \mathrm{GHz}$ are likely too low, which gives a strange behavior. For clarification, Fig. 2 presents the $I L$ characteristics for each cable type defined by the ITU based on the attenuation vs frequency table presented in [15]. A $100 \mathrm{~m}$ long cable is used as reference. It can be observed that the $I L$ characteristics are not well dimensioned in some regions of the spectrum, especially for the RG-6 and RG- 11 cables. Thus, a cable model fitted to these data does not lead to reliable results.

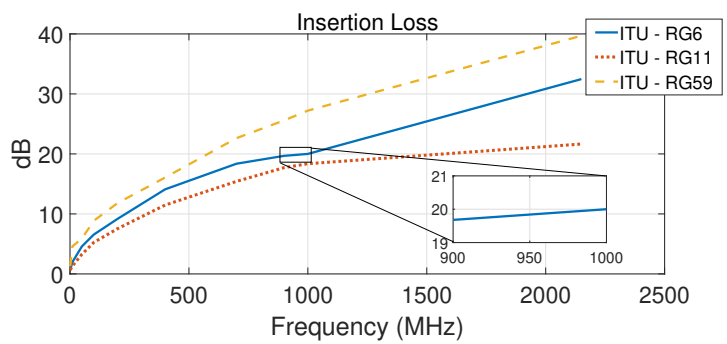

Fig. 2. Insertion loss characteristics for a $100 \mathrm{~m}$ long cable derived from the signal loss vs frequency table presented in [15]. Considering the RG-6, there is a difference of $0.32 \mathrm{~dB}$ between $900 \mathrm{MHz}$ and $1 \mathrm{GHz}$.

In order to avoid this situation, we fitted the KM1 cable model parameters to some coaxial lines manufactured by CommScope using their datasheets as basis [19]. The CommScope lines were selected according with their similarity with respect to the ones standardized by the ITU and more information about them can be found in Table I. In this way, Table II presents the KM1 model parameters values fitted to the CommScope RG-6, RG-59 and RG-11 cables and Fig. 3 shows the resulting $I L$ curves. It can be noted a better balance between the $I L$ attenuation behavior among the different cables.

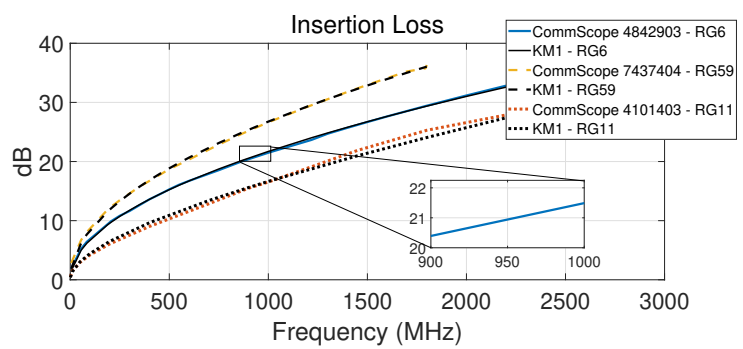

Fig. 3. Insertion loss characteristics for a $100 \mathrm{~m}$ long cable derived from the KM1 cable model fitted to the CommScope datasheets. Considering the RG-6, there is a difference of $1.1 \mathrm{~dB}$ between $900 \mathrm{MHz}$ and $1 \mathrm{GHz}$.

An absolute error of $\approx 0.5 \mathrm{~dB}$ between the data provided by the datasheets and the model estimates is observed for all cases up to $1 \mathrm{GHz}$. 
TABLE I

INFORMATION ABOUT THE CABLES MANUFACTURED BY COMMSCOPE USED IN THE TESTS [19]. PE $=$ POLYETHYLENE, PVC $=$ POLYVINYL CHLORIDE, FEP $=$ FLUORINATED ETHYLENE PROPYLENE.

\begin{tabular}{ccccc} 
& \multicolumn{2}{c}{ RG-6 } & RG-59 & RG-11 \\
\hline Product identification & 4842903 F677TSEF XP & 7437404 F59 HEC VV XP BLK & 4101403/10|2285V WTRL \\
Application/Category & Drop cable & Headended cable & Videstellite cable \\
Center conductor material & Copper-clad steel & Silver-plated copper-clad steel & 1.628 \\
Diameter over center conductor $(\mathrm{mm})$ & 1.016 & 0.813 & 7.214 \\
Diameter over inner shield (tape) $(\mathrm{mm})$ & 4.75 & 3.835 & Foam FEP \\
Dielectric material & Foam PE & PE & PVC \\
Jacket material & PE & Fire retardant PVC & 52.49 \\
Capacitance $-C(\mathrm{pF} / \mathrm{m})$ & 53.15 & - & 75 & 86 \\
Characteristic impedance $-Z_{0}(\Omega)$ & 75 & 85 & Plenum \\
Velocity of propagation $(\%)$ & 85 & Indoor & \\
Environmental space & Buried & & \\
\hline
\end{tabular}

TABLE II

KM1 CABLE MODEL PARAMETERS FITTED TO THE COMMSCOPE RG-6, RG-59 AND RG-11 CABLES.

\begin{tabular}{ccc} 
& $k_{1}$ & $k_{2}$ \\
\hline RG-6 & 0.6697 & 0.0005321 \\
RG-59 & 0.8356 & 0.0003333 \\
RG-11 & 0.407 & 0.003775 \\
\hline
\end{tabular}

The parameters values presented in Table II model the $I L$ in $\mathrm{dB}$ scale and were fitted using a reference length of $100 \mathrm{~m}$ and a frequency grid in $\mathrm{MHz}$. In order to express these parameters in terms of the attenuation coefficient, which is often given in nepers, as well as adopt other reference lengths, like in [16], proper scaling must be performed. In this way, one can use the following relation to scale from $\mathrm{dB} / 100 \mathrm{~m}$ to $\mathrm{Np} / 100 \mathrm{~m}$ : $I L(f)(\mathrm{dB} / 100 \mathrm{~m})=8.686 \times 100 \times \alpha(f)$, where $\alpha(f)$ is the cable's frequency dependent attenuation coefficient (given in nepers per meter) and $f$ is the frequency in $\mathrm{MHz}$ [20].

\section{MGfast System Model and Simulation PARAMETERS}

\section{A. Transmission Capacity}

In order to estimate the data capacity of MGfast access systems over coaxial cables, PSD-based simulations were performed in STDD operation mode. Therefore, assuming that the coaxial media is a memoryless additive white Gaussian noise (AWGN) channel and considering that MGfast uses DMT [4], [21], in which a serial data stream is divided into multiple orthogonal independent sub-channels (also called tones) $k$ containing a fixed bandwidth $\Delta f$, the bit allocation, i.e., the bit loading, in each sub-channel $k$ is obtained from:

$$
b_{k}=\left\lfloor\log _{2}\left(1+\frac{\left|H_{k}\right|^{2} \rho_{k}}{\Gamma \sigma_{k}}\right)\right\rfloor,
$$

where $\Gamma$ is the signal-to-noise ratio (SNR) gap, which is a function of the line code, desired noise margin and probability of symbol error ${ }^{2}$ [22], [23], and $H_{k}, \rho_{k}$ and $\sigma_{k}$ denote the transfer function, the transmit PSD and the background noise experienced in sub-channel $k$, respectively.

\footnotetext{
${ }^{2}$ The SNR gap indicates how close the bit rate reaches the theoretical best performance [22]. For example, a gap of $\Gamma=1(0 \mathrm{~dB})$ leads to the channel capacity [23].
}

In the DMT modulator, the bit allocation in each subchannel guides a constellation mapper block whose outputs are several quadrature amplitude modulation (QAM), even quadrature phase shift keying (QPSK), symbols [21], [22]. One QAM/QPSK symbol is created per tone, and they are, in turn, grouped to create the so-called DMT symbol. Thus, the data rate achieved by a given MGfast user is obtained by the summation of the bit allocation in each tone (that gives the total number of bits per DMT symbol) times the DMT symbol rate, $R_{d m t}$. If no overhead is taken into account, the DMT symbol rate equals $\Delta_{f}$ and the data rate is obtained by:

$$
R=\Delta_{f} \sum_{k} b_{k} .
$$

In Equations (3) and (4), the transfer function is derived from the $I L$ equation defined by the KM1 cable model discussed in Subsection II-B. The background noise is assumed to be $-150 \mathrm{dBm} / \mathrm{Hz}$ [3] and, since crosstalk has no significant impact on coaxial cables (at least up to the frequencies considered in this work), it is assumed that the ingress effects from neighbors lines are smaller than the background noise [14], [24], [25]. The tone spacing $\Delta_{f}$ follows [11] and is equal to $51.75 \mathrm{kHz}$. For the SNR gap, it is adopted $\Gamma=10.75 \mathrm{~dB}$, which incorporates a $5 \mathrm{~dB}$ coding gain and a $6 \mathrm{~dB}$ margin [21]. The SNR gap is typically specified in $\mathrm{dB}$ and converted to linear scale using $10^{\left(\frac{\Gamma}{10}\right)}$ to be used in Equation (3).

The number of bits per DMT tone, $b_{k}$, varies along the transmission band according with the SNR and ranges from a minimum to a maximum level ${ }^{3}$, such that $b_{k}^{\min } \leq b_{k} \leq$ $b_{k}^{\max }, \forall k$. This paper follows [11] and assumes $b_{k}^{\min }=2$. Regarding the maximum number of bits per sub-channel, $b_{k}^{\max }$, two values were tested: 12 and 14 bits, which allows using modulation orders as high as 4096 and 16384 QAM, respectively. In fact, both the latest G.fast standard and the new MGfast specification give support to 12 and 14 bits per DMT tone [11], [12]. Employing $b_{k}^{\max }=12$ bits is suitable for G.fast loops [26] (e.g., up to $250 \mathrm{~m}$ ), whereas using a number of bits lower than 12 may lead to a reduction on the system performance. Actually, $b_{k}^{\max }=12$ bits is a convenient

\footnotetext{
${ }^{3}$ A major difference between DMT and other multi-carrier modulation schemes, like orthogonal frequency division multiplexing (OFDM), is that DMT adopts a variable number of bits per sub-channel, whereas OFDM uses a fixed number of bits per tone [23].
} 
approach considering typical restrictions in the analog frontend, for example, due to the limited resolution of the analogto-digital converter [3], [5]. For short loops, on the other hand, such as the ones planned to be used in MGfast (e.g., up to $100 \mathrm{~m}$ ), a noticeable data rate increase is observed assuming $b_{k}^{\max }=14$ bits [27].

It is important to highlight that Equation (4) does not consider any overhead originated from maneuvers to make the system cope with the channel impairments in time domain. For example, in real deployments, both the propagation delay and delay spread in the coaxial cables will reduce the capacity of MGfast systems [5], [6]. These quantities impact on the dispersion of a DMT symbol in another adjacent DMT symbol, leading to the so-called inter-symbol interference (ISI). In order to minimize this drawback, DMT-based systems adopt a cyclic prefix. The cyclic prefix aims at compensating the channel dispersion as well as softening the effect of reflections caused by impedance mismatches at the ends of the loops [28]. It consists in copying $L_{c p}$ samples from the end of the DMT symbol to its beginning (creating a prefix), before sending the time-domain samples to the digital-to-analog chip. Another important benefit of using the cyclic prefix is to avoid inter-carrier interference (ICI) between tones of the same DMT symbol, thus, ensuring tone orthogonality [9], [29], [30]. Unfortunately, the cyclic prefix adds some overhead to the system, which, in turn, must be calculated. Hence, considering a cyclic prefix composed by $L_{c p}$ samples, the resulting data rate is given by:

$$
R=\Delta_{f}\left(\frac{N}{N+L_{c p}}\right) \sum_{k} b_{k}
$$

where $N$ is the size of the inverse discrete Fourier transform (IDFT)/discrete Fourier transform (DFT) blocks employed in the DMT modulator/demodulator [21], [22], respectively. The achievable data rate is affected by the cyclic prefix since the DMT symbol rate is reduced as a result of the inclusion of such guard interval. In this regards, if $L_{c p}=0$ the DMT symbol rate equals $\Delta_{f}$.

As mentioned, the cyclic prefix has to be the same length as the channel impulse response [29]. In fact, in STDD MGfast systems, the cyclic prefix only has to counter the impulse response delay spread, whereas in full-duplex MGfast the cyclic prefix is used to counter the delay spread and a cyclic suffix is employed to counter the propagation delay in the cable [5], [6]. This paper adopts cyclic prefix configurations compatible with G.fast [4], which include an associated overhead to the system varying between 0 and 13\% [3], [5], [31].

\section{B. Deployments}

The simulations analyze deployments in which fiber lines are taken up to the last distribution point/tap in the hybrid fiber-coaxial (HFC) network [11], [25], [32]-[34]. From this location, point-to-point (P2P) connection using coaxial cables are used to establish the communication between the DPU and CPE. The cable types include the RG-6, RG-11 and RG-59 standards discussed in Subsection II-B and the loop lengths range from $10 \mathrm{~m}$ up to $100 \mathrm{~m}$. The evaluations follow the specifications for a typical deployment of P2P MGfast in MDU without SAT TV sharing the coaxial medium [11]. It is assumed that there are no splitters, bridge taps and in-line amplification devices in the MGfast path. Fig. 4 depicts the analyzed deployments.

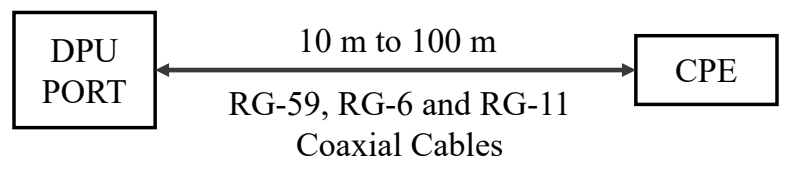

Fig. 4. Deployment of P2P MGfast in MDU without SAT TV service sharing the coaxial cables, as in [11].

\section{MGfast Profiles, PSD Masks and Maximum Transmit Pow- ers}

The lowest frequency considered to be used in MGfast is 2.2 MHz. Thus, in order to analyze the MGfast transmission profiles defined in [11], as well as exploit even larger bandwidths, the simulations cover frequency bands from $2.2 \mathrm{MHz}$ up to $424 \mathrm{MHz}, 848 \mathrm{MHz}$ and $1696 \mathrm{MHz}$. However, since the G.mgfast recommendation is not completely finished, some features are still open for discussion. For example, in terms of the PSD mask, only the MGfast $424 \mathrm{MHz}$ profile has a defined one [4], [11], [13]. It is compatible with the G.fast standard below $212 \mathrm{MHz}(-65 \mathrm{dBm} / \mathrm{Hz}$ below $30 \mathrm{MHz}$, decreases to $-73 \mathrm{dBm} / \mathrm{Hz}$ at $30 \mathrm{MHz}$, drops to $-76 \mathrm{dBm} / \mathrm{Hz}$ at $106 \mathrm{MHz}$ and reduces to $-79 \mathrm{dBm} / \mathrm{Hz}$ at $212 \mathrm{MHz}$ ). From $212 \mathrm{MHz}$ to $424 \mathrm{MHz}$ it assumes a $-79 \mathrm{dBm} / \mathrm{Hz}$ level [4].

For composing the PSD mask of the MGfast $848 \mathrm{MHz}$ profile, this work considers a linear decrease of $6 \mathrm{~dB}$ from $424 \mathrm{MHz}$ to $848 \mathrm{MHz}$, leading the PSD to a $-85 \mathrm{dBm} / \mathrm{Hz}$ level. The process is similar to that one used to define the PSD mask of the $424 \mathrm{MHz}$ profile. The PSD associated with the MGfast $1696 \mathrm{MHz}$ profile is obtained extending the -85 $\mathrm{dBm} / \mathrm{Hz}$ level from $848 \mathrm{MHz}$ to $1696 \mathrm{MHz}$. Fig. 5 shows the PSD mask used in the MGfast $424 \mathrm{MHz}$ profile extended to impose restrictions on the MGfast $848 \mathrm{MHz}$ and $1696 \mathrm{MHz}$ profiles $^{4,5}$.

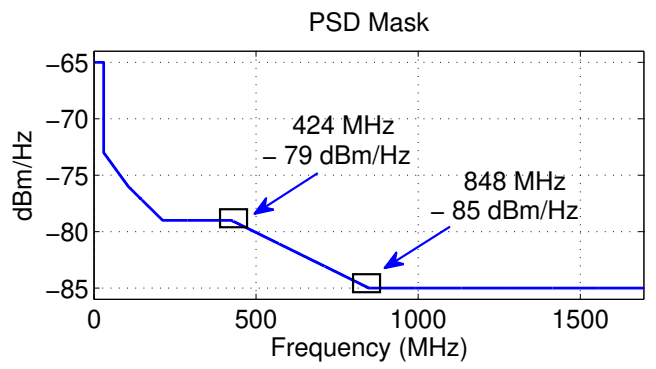

Fig. 5. PSD mask used in the MGfast $424 \mathrm{MHz}$ profile extended to impose restrictions on the MGfast $848 \mathrm{MHz}$ and $1696 \mathrm{MHz}$ profiles.

\footnotetext{
${ }^{4} \mathrm{~A}$ deeper analysis for validating the PSD masks used in the $848 \mathrm{MHz}$ and $1696 \mathrm{MHz}$ profiles, taking as reference norms like the CISPR 22 [35], is out of the scope of this paper.

${ }^{5}$ In practical deployments, there could be a need for further reductions or notches in sensitive bands, for example, to protect Global Positioning System (GPS) frequencies in case of the $1696 \mathrm{MHz}$ profile.
} 
Regarding the maximum aggregate transmit power, in both downstream and upstream, the recommendation suggests +2 $\mathrm{dBm}$ for the MGfast $424 \mathrm{MHz}$ profile operating over coaxial cables [11]. For the $848 \mathrm{MHz}$ profile, and for the possible $1696 \mathrm{MHz}$ one, such values remain undefined. Due to this fact, this work assumes $+4 \mathrm{dBm}$ for the $848 \mathrm{MHz}$ profile and two options for the $1696 \mathrm{MHz}$ profile: $+6 \mathrm{dBm}$ and +8 $\mathrm{dBm}$. It is important to emphasize that a maximum aggregate transmit power of $+8 \mathrm{dBm}$ is already defined for G.fast systems operating over twisted-pair cables [12].

\section{Power Allocation}

The PSD mask, $\rho_{k}^{\text {mask }}$, defines the maximum transmit level at which each DMT tone is allowed to transmit, such that: $0 \leq \rho_{k} \leq \rho_{k}^{\text {mask }}, \forall k$. Nevertheless, it is important to note that lower PSD levels can potentially give the same bit allocation as that ones provided by the PSD mask, with benefits of using lower power and keeping the maximum number of bits per sub-channel, $b_{k}^{\max }$, unchanged. In this context, this work assumes that the MGfast transceivers set their transmit PSDs using the following equation:

$$
\rho_{k}=\left(2^{b_{k}^{\text {mask }}}-1\right) \frac{\Gamma \sigma_{k}}{\left|H_{k}\right|^{2}},
$$

where $b_{k}^{\text {mask }}=\min \left(b_{k}^{\text {max }}, b_{k}\left(\rho_{k}^{\text {mask }}\right)\right)$. Equation (6) assumes a finite granularity at each frequency point in the bit distribution and gives the necessary amount of power (in terms of PSD level) for achieving a target number of bits per DMT tone in any part of the MGfast spectrum, considering a given fixed $\Gamma$, e.g., $10.75 \mathrm{~dB}$.

\section{Simulation Results}

This Section presents the results. Numbers related to the maximum achievable data rates and associated transmit powers are presented first. In the end, data rates obtained using distinct cyclic prefix configurations are exposed.

\section{A. Maximum Achievable Data Rates}

Figs. 6, 7 and 8 present the maximum achievable data rates considering the three MGfast profiles discussed in Subsection III-C. The tests adopt the RG-11, RG-6 and RG-59 coaxial cable types for connecting the DPU to the CPE and the loops range from $10 \mathrm{~m}$ to $100 \mathrm{~m}$.

It is possible to observe from Fig. 6 that an aggregate data rate of at least $5 \mathrm{Gbps}$ is achieved using the MGfast $424 \mathrm{MHz}$ profile. Besides, for this specific case, the type of the coaxial cable used to connect the DPU to the CPE does not affect the results. Since the SNR is (relatively) not much degraded in this narrower bandwidth, the line standard does not impact on the achievable rates.

Regarding the MGfast $848 \mathrm{MHz}$ profile, it can be noted from Fig. 7 that an aggregate data rate of at least $10 \mathrm{Gbps}$ is achieved in most of the cases. The outlier is related to the RG59 cable. Due to its higher attenuation, as the distance between the DPU and the CPE increases, it becomes unable to hold the referred data rate. This behavior can be observed for distances

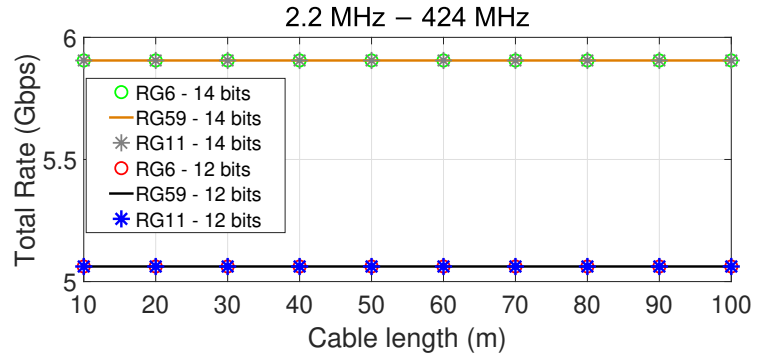

Fig. 6. Maximum achievable data rates for the MGfast $424 \mathrm{MHz}$ profile assuming $b_{k}^{\max }=12,14$. It is possible to observe that for this specific profile, the type of the coaxial cable used to connect the DPU to the CPE does not affect the results.

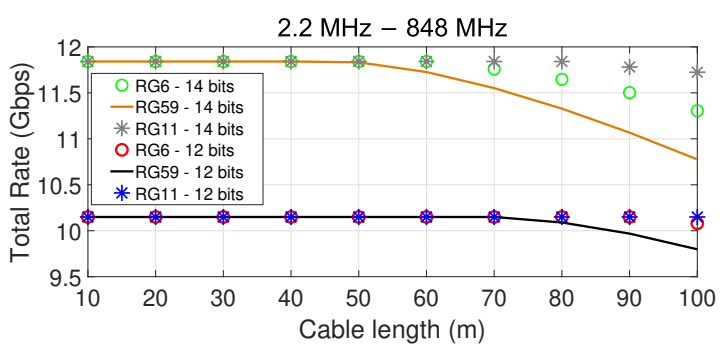

Fig. 7. Maximum achievable data rates for the MGfast $848 \mathrm{MHz}$ profile assuming $b_{k}^{\max }=12,14$.

longer than $87 \mathrm{~m}$ adopting $b_{k}^{\max }=12$. Considering $b_{k}^{\max }=$ 14 , the data rates rise even more and can reach up to 11.8 Gbps in short distances, which represents an increase of about $18 \%$. In general, for $b_{k}^{\max }=12$ the type of the cable does not affect the data capacity when the distances are shorter than $70 \mathrm{~m}$, whereas for $b_{k}^{\max }=14$ the same behavior is observed from cables shorter than $50 \mathrm{~m}$. These numbers corroborate that for FTTB and FFTdp deployments, even a low-performance cable as the RG-59 can reach the same results obtained with a high-performance one such as the RG-11.

Fig. 8 shows the results for the MGfast $1696 \mathrm{MHz}$ profile. It can be observed that the achievable data rates can be as high as $20.3 \mathrm{Gbps}$ and $23.7 \mathrm{Gbps}$, depending on the chosen $b_{k}^{\max }$. However, the data rates decrease more sharply than the MGfast $848 \mathrm{MHz}$ profile as the distance between the DPU and the CPE increases. For example, considering the RG-59 line and $b_{k}^{\max }=14$, there is a reduction from $23.7 \mathrm{Gbps}$ to about 17.1 Gbps when the cable length increases from $30 \mathrm{~m}$ to 100 $\mathrm{m}$. Moreover, the distance in which the cable type does not affect the achievable data rates is also reduced. For $b_{k}^{\max }=12$, distances shorter than $50 \mathrm{~m}$ exhibit this behavior, whereas for $b_{k}^{\max }=14$ this occurs for distances shorter than $30 \mathrm{~m}$.

The MGfast data rates presented in Figs. 6, 7 and 8 were calculated from bit loading estimates, following the system model exposed in Section III. Hence, for completeness, we present some results in terms of the bit loadings. Fig. 9 exhibits the bit loading calculated using the MGfast $1696 \mathrm{MHz}$ profile in conjunction with RG-59 cables of distinct lengths and $b_{k}^{\max }=12,14$. It can be noted the typical reduction on the number of bits as the frequency increases, which is caused by the SNR degradation. For long cables, only 6 bits are allowed to be allocated in the DMT sub-channels placed in the end of 


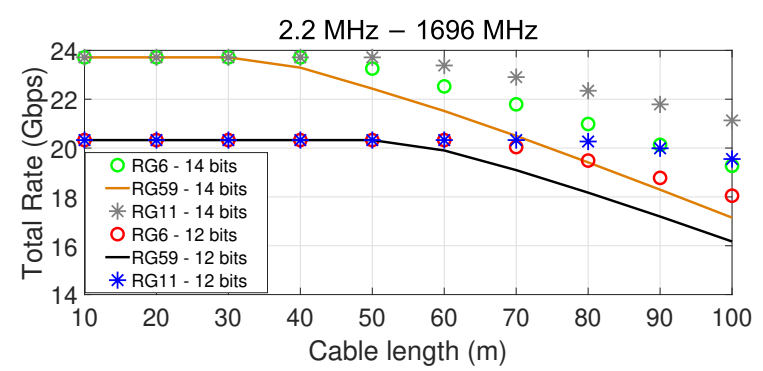

Fig. 8. Maximum achievable data rates for the MGfast $1696 \mathrm{MHz}$ profile assuming $b_{k}^{\max }=12,14$.

the band. Similarly, Fig. 10 exhibits the bit loading estimated using the MGfast $1696 \mathrm{MHz}$ profile in conjunction with RG-6 cables. The bit loading estimates for the other MGfast profiles and cables follow the pattern depicted in Fig. 9 and Fig. 10 and will not be presented in the sequence for convenience.

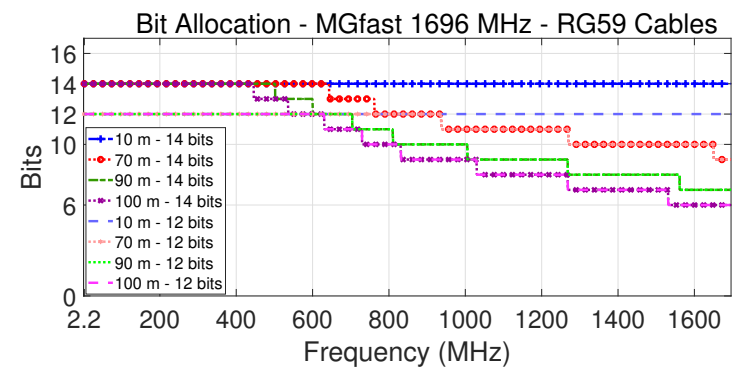

Fig. 9. Bit loading for the MGfast $1696 \mathrm{MHz}$ profile using RG-59 cables of distinct lengths and $b_{k}^{\max }=12,14$.

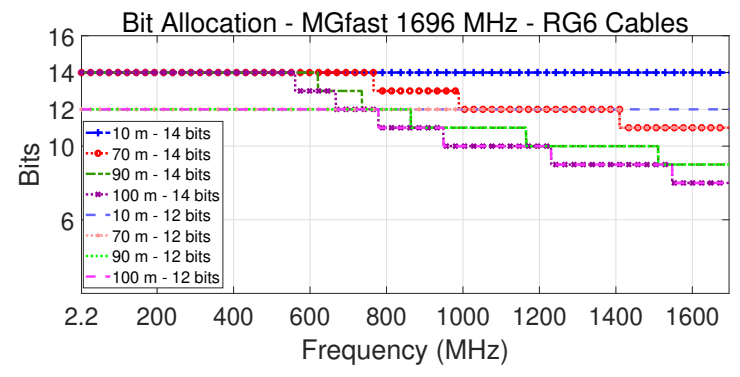

Fig. 10. Bit loading for the MGfast $1696 \mathrm{MHz}$ profile using RG-6 cables of distinct lengths and $b_{k}^{\max }=12,14$.

\section{B. Transmit Powers}

Fig. 11 presents the transmit powers associated with the maximum achievable data rates for the MGfast $424 \mathrm{MHz}$ profile. The upper plot shows the results considering $b_{k}^{\max }=12$, while the lower one shows the results for $b_{k}^{\max }=14$. It can be observed that the $+2 \mathrm{dBm}$ transmit power limitation is always sustained for this specific profile assuming $b_{k}^{\max }=12$. Assuming $b_{k}^{\max }=14$, the maximum limit of $+2 \mathrm{dBm}$ is exceeded only for loops in the range of $100 \mathrm{~m}$.

Fig. 12 shows the results for the MGfast $848 \mathrm{MHz}$ profile. Similarly, the upper plot shows the results considering $b_{k}^{\max }=$ 12 , while the lower one shows the results for $b_{k}^{\max }=14$.
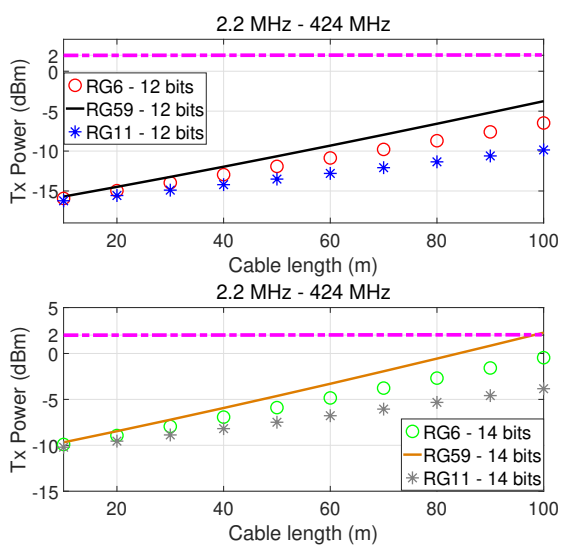

Fig. 11. Transmit powers for the MGfast $424 \mathrm{MHz}$ profile assuming $b_{k}^{\max }=$ 12 (upper plot) and $b_{k}^{\max }=14$ (lower plot). The maximum limit of $+2 \mathrm{dBm}$ is maintained in most of the cases.
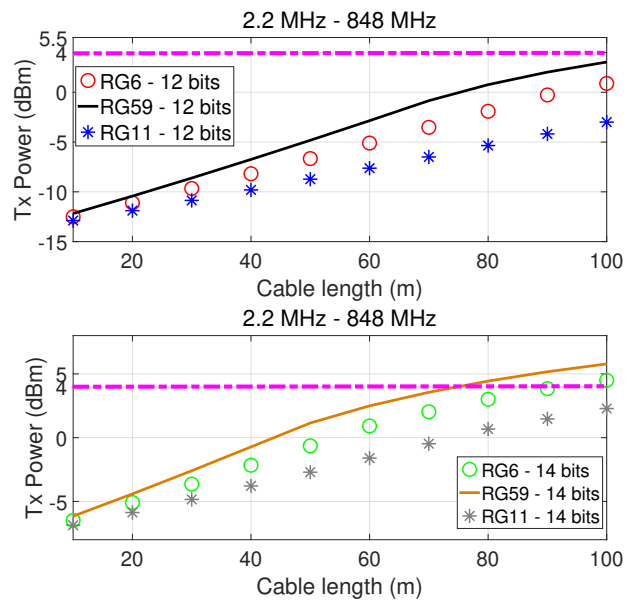

Fig. 12. Transmit powers for the MGfast $848 \mathrm{MHz}$ profile assuming $b_{k}^{\max }=$ 12 (upper plot) and $b_{k}^{\max }=14$ (lower plot). The maximum limit of $+4 \mathrm{dBm}$ is maintained in most of the cases.

Assuming $b_{k}^{\max }=12$, the highest transmit power belongs to the $100 \mathrm{~m}$ long RG-59 cable, which is about $+3 \mathrm{dBm}$. Considering a high-performance line, like the RG-11, the transmit power is lower than $-2.9 \mathrm{dBm}$, which is still too small if compared to the $+4 \mathrm{dBm}$ limit previously defined for the $848 \mathrm{MHz}$ profile. Despite of this, when $b_{k}^{\max }=14$, the transmit powers can exceed the power restrictions. For example, considering the RG-59 cable, the maximum limit of $+4 \mathrm{dBm}$ is exceeded for loops longer than about $75 \mathrm{~m}$. Similarly, such a limit is also extrapolated for loops longer than 92 m considering the RG-6 cable.

The results from this controlled test indicate that for the MGfast $848 \mathrm{MHz}$ profile, a power-efficient implementation is always achieved if $b_{k}^{\max }=12$, whereas high modulation orders present viability only for cables shorter than $75 \mathrm{~m}$. Note that, in real implementations, it might be possible to use $b_{k}^{\max }=14$ bits on low frequencies and $b_{k}^{\max }=12$ bits (or fewer) on high frequencies in order to fit the power budget, however, such analysis is not evaluated here (the maximum number of bits per tone is the same for the whole band).

Fig. 13 exhibits the results for the MGfast $1696 \mathrm{MHz}$ profile. In this case, the maximum transmit power restriction 
of $+8 \mathrm{dBm}$ is always respected. It can be noted that even using a higher modulation order, i.e., $b_{k}^{\max }=14$, the transmit power is below $+8 \mathrm{dBm}$. On the other hand, the maximum limit of $+6 \mathrm{dBm}$ is overcame in some cases. For example, assuming $b_{k}^{\max }=14$, the limit is exceeded considering RG-59 cables longer than about $65 \mathrm{~m}$ and RG-6 lines longer than $80 \mathrm{~m}$.
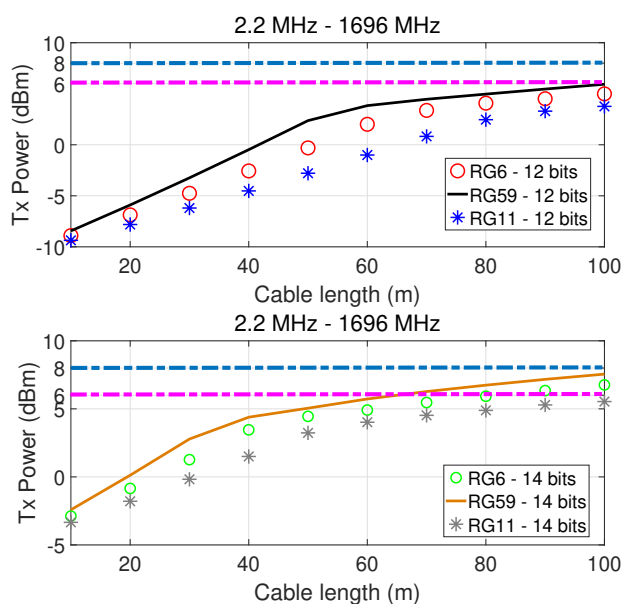

Fig. 13. Transmit powers for the MGfast $1696 \mathrm{MHz}$ profile assuming $b_{k}^{\max }=12$ (upper plot) and $b_{k}^{\max }=14$ (lower plot). The maximum limit of $+8 \mathrm{dBm}$ is always maintained. However, the maximum limit of $+6 \mathrm{dBm}$ is overcame in some cases.

\section{Data Rates Obtained Using Distinct Cyclic Prefix Config- urations}

The maximum achievable data rate estimated for a given channel condition, SNR gap and transmit PSD is an important quantity, however, in real implementations the cyclic prefix must be taken into account, hence, Fig. 14 presents the aggregate net data rates estimated using distinct cyclic prefix configurations for the MGfast $424 \mathrm{MHz}$ profile and $b_{k}^{\max }=14$. In the legend, $T_{c p}$ stands for the cyclic prefix period. The numbers corroborate that for this specific profile the results are not influenced by the coaxial cable type used in the access network. Furthermore, a 5 Gbps aggregate net data rate is still achievable even in a configuration in which a cyclic prefix containing 2560 samples is adopted.

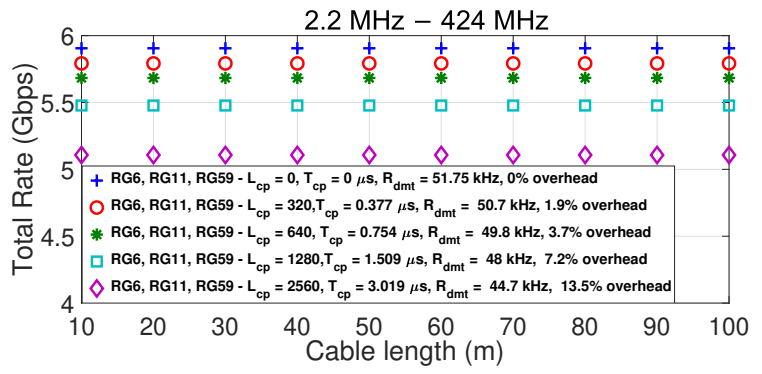

Fig. 14. Data rates for the MGfast $424 \mathrm{MHz}$ profile assuming $b_{k}^{\max }=14$, RG-6, RG-11 and RG-59 cables and distinct cyclic prefix configurations.

Continuing, Figs. 15, 16 and 17 exhibit the results for the $848 \mathrm{MHz}$ profile and $b_{k}^{\max }=14$. In this case, the type of cable influences in the estimates. It is possible to observe that even in the worst-case, derived from the RG-59 cable, a data rate higher than 9 Gbps is reached using a DMT symbol rate lower than $48 \mathrm{kHz}$. In addition to this, data rates of at least $10.5 \mathrm{Gbps}$ are achieved using a configuration that leads to an overhead of about $1.9 \%$, like in [5].

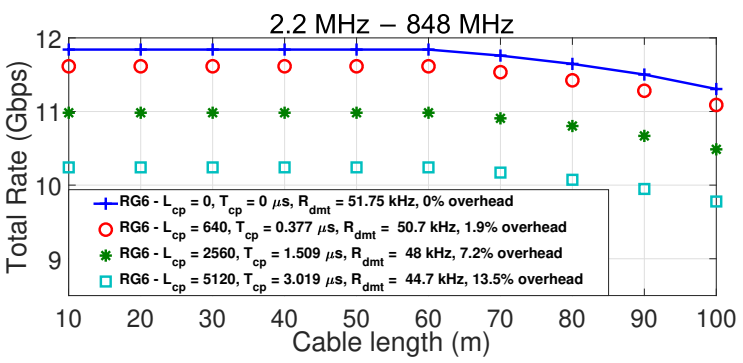

Fig. 15. Data rates for the MGfast $848 \mathrm{MHz}$ profile assuming $b_{k}^{\max }=14$, RG-6 cables and distinct cyclic prefix configurations.

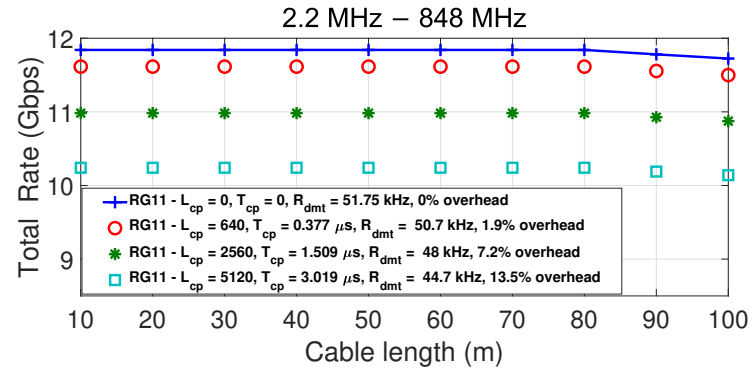

Fig. 16. Data rates for the MGfast $848 \mathrm{MHz}$ profile assuming $b_{k}^{\max }=14$, RG-11 cables and distinct cyclic prefix configurations.

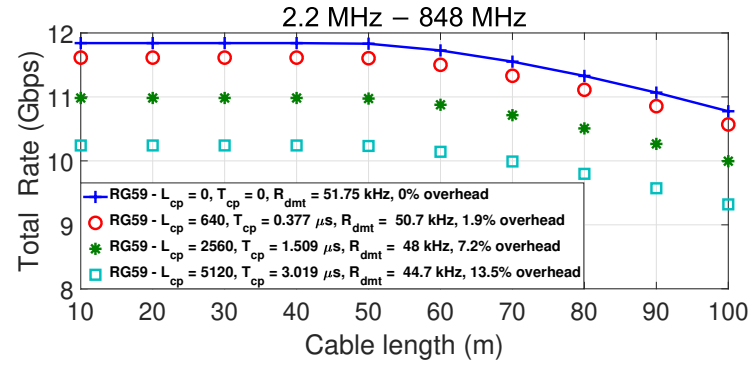

Fig. 17. Data rates for the MGfast $848 \mathrm{MHz}$ profile assuming $b_{k}^{\max }=14$, RG-59 cables and distinct cyclic prefix configurations.

At last, Figs. 18, 19 and 20 exhibit the results for the $1696 \mathrm{MHz}$ profile and $b_{k}^{\max }=14$. A data rate higher than 14 Gbps is reached in the worst-case, related to the RG-59 cable, presented in Fig. 20. This occurs when the system uses a cyclic prefix period of about $3 \mu$ s, which leads to a DMT symbol rate lower than $48 \mathrm{kHz}$ and almost $13 \%$ of associated overhead. In fact, a data rate of at least 20 Gbps is sustained in almost half of the analyzed scenarios, highlighting the potential of MGfast systems to enable multigigabit transmission on existing copper cables.

\section{CONClusions}

MGfast is a new standard from the ITU that aims at achieving data rates as high as tens of Gbps using metallic 


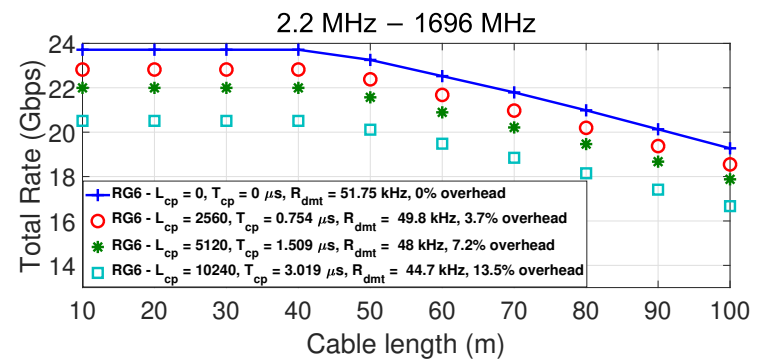

Fig. 18. Data rates for the MGfast $1696 \mathrm{MHz}$ profile assuming $b_{k}^{\max }=14$, RG-6 cables and distinct cyclic prefix configurations.

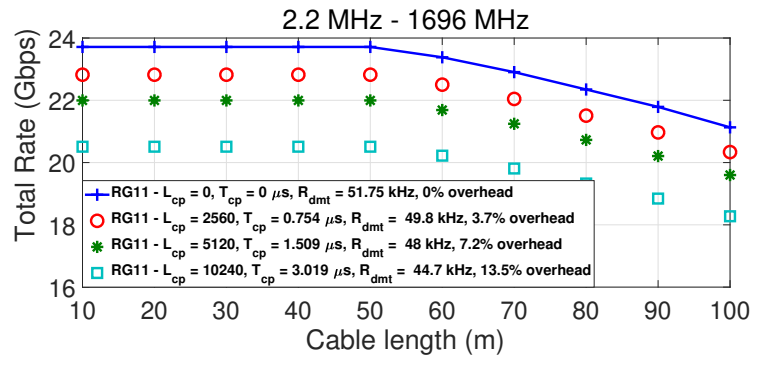

Fig. 19. Data rates for the MGfast $1696 \mathrm{MHz}$ profile assuming $b_{k}^{\max }=14$, RG-11 cables and distinct cyclic prefix configurations.

cables designed for POTS and TV services. In this work, the performance of MGfast systems based on coaxial cables was verified through PSD-based simulations, assuming the STDD operation mode and modulation schemes like 4096 and 16384 QAM per DMT sub-channel. It was observed that MGfast over coaxial cables can achieve data rates as high as 23 Gbps using loop lengths compatible with FTTdp and FTTB deployments. For short loops, where attenuation does not significantly impact on rate percentage, the results show that the same data rate can be achieved by both high and low performance cables. The tests considered a variety of configurations for the cyclic-prefix length and associated DMT symbol rate. Regarding the transmit powers, most of the analyzed cases respected the maximum limits defined in the draft version of the standard. Thus, in general, the results showed that the MGfast based on coaxial cables can be an access technology aggregating high capacity and energy efficiency.

\section{REFERENCES}

[1] W. Coomans, R. B. Moraes, K. Hooghe, A. Duque, J. Galaro, M. Timmers, A. J. van Wijngaarden, M. Guenach, and J. Maes, "XG-FAST: Towards $10 \mathrm{~Gb} / \mathrm{s}$ copper access," in 2014 IEEE Globecom Workshops (GC Wkshps), Dec. 2014, pp. 630-635, doi: 10.1109/GLOCOMW.2014.7063503.

[2] — "XG-fast: the 5th generation broadband," IEEE Commun. Mag., vol. 53, no. 12, pp. 83-88, Dec. 2015, doi: 10.1109/MCOM.2015.7355589.

[3] R. Strobel, "Copper transmission for multi-gigabit hybrid copper-fiber access networks," in 2017 Int. Workshop on Fiber Opt. in Access Netw. (FOAN), Nov. 2017, pp. 1-6, doi: 10.1109/FOAN.2017.8215256.

[4] V. Oksman, R. Strobel, T. Starr, J. Maes, W. Coomans, M. Kuipers, E. B. Tovim, and D. Wei, "MGFAST: A New Generation of Copper Broadband Access," IEEE Commun. Mag., vol. 57, no. 8, pp. 14-21, Aug. 2019, doi: 10.1109/MCOM.2019.1800844.

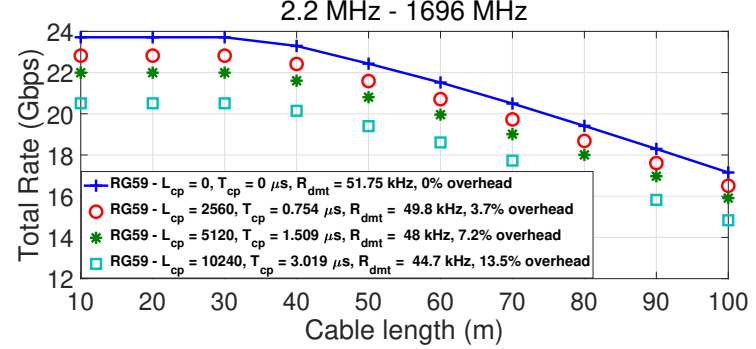

Fig. 20. Data rates for the MGfast $1696 \mathrm{MHz}$ profile assuming $b_{k}^{\max }=14$, RG-59 cables and distinct cyclic prefix configurations.

[5] R. Strobel, "Physical layer framework for the multi-gigabit copper-fiber access networks," Fiber and Integr. Opt., vol. 37, no. 6, pp. 291-313, 2018, doi: 10.1080/01468030.2018.1523507.

[6] P. Tsiaflakis, Y. Lefevre, W. Coomans, and J. Maes, "Friendly Full Duplex: A Multi-User Full Duplex Method for MGfast in Coexistence with G.fast," in 2018 IEEE Global Commun. Conf. (GLOBECOM), 2018, pp. 1-6, doi: 10.1109/GLOCOM.2018.8647579.

[7] O. Lamparter, L. Fang, J. Bischoff, M. Reitmann, R. Schwendener, T. Zasowski, and X. Zhang, "Multi-gigabit over copper access networks: Architectural evolution and techno-economic analysis," IEEE Commun. Mag., vol. 57, no. 8, pp. 22-27, 2019, doi: 10.1109/MCOM.2019.1800847.

[8] J. Verdyck, W. Lanneer, P. Tsiaflakis, W. Coomans, P. Patrinos, and M. Moonen, "Optimal dynamic spectrum management algorithms for multi-user full-duplex dsl," IEEE Access, vol. 7, pp. 106600-106616, 2019, doi: 10.1109/ACCESS.2019.2926616.

[9] Y. Zhang, R. Zhang, J. Zhang, T. Bai, A. F. Al Rawi, M. Moonen, and L. Hanzo, "Far-end crosstalk mitigation for future wireline networks beyond g.mgfast: A survey and an outlook," IEEE Access, vol. 8, pp. 9998-10 039, 2020, doi: 10.1109/ACCESS.2019.2963571.

[10] S. M. Zafaruddin and A. Leshem, "Multi-gigabit wireline systems over copper: An interference cancellation perspective," arXiv: Signal Process., 2020, [Online], Available: https://arxiv.org/abs/2005.08264.

[11] Editor G.mgfast, "Draft text for G.mgfast-PHY," ITU-T contribution, Temporary Document SG15 - TD329/WP1, Jul. 2019.

[12] ITU-T G.9701, "Recommendation ITU-T G.9701," Fast access to subscriber terminals (FAST) - Physical layer specification, Mar. 2019.

[13] ITU-R WP1A, "Recent developments concerning coexistence of wired telecommunication with radiocommunication systems," ITU-R document, 1A/327 - E, May 2018.

[14] Proximus, "G.fast: Proximus requirements for G.fast operation on coax and CAT5 wires up to at least 150 meters," ITU-T contribution, COM 15 - C 1840 - E, Feb. 2016.

[15] ADTRAN, AT\&T, Sckipio, "G.fast: Update of coax cable configurations working text for Annex X," ITU-T contribution, COM 15 - C 2152 E, Sep. 2016.

[16] D. Acatauassu, S. Höst, C. Lu, M. Berg, A. Klautau, and P. O. Börjesson, "Simple and causal twisted-pair channel models for G.fast systems," in 2013 IEEE Global Commun. Conf. (GLOBECOM), Dec. 2013, pp. 2834-2839, doi: 10.1109/GLOCOM.2013.6831504.

[17] — " "Simple and causal copper cable model suitable for G.fast frequencies," IEEE Trans. on Commun., vol. 62, no. 11, pp. 4040-4051, Nov. 2014, doi: 10.1109/TCOMM.2014.2364585.

[18] D. Acatauassu, S. Höst, C. Lu, M. Berg, J. Costa, A. Klautau, P. Ödling, and P. O. Börjesson, "KHM cable model parameters for ITU-T G.fast reference loops," in 2015 IEEE Conf. on Standards for Commun. and Netw. (CSCN), Oct. 2015, pp. 168-173, doi: 10.1109/CSCN.2015.7390439.

[19] CommScope, "CommScope Website," [Online], Available: http://www.commscope.com.

[20] J.-J. Werner, "The HDSL environment (high bit rate digital subscriber line)," IEEE J. on Sel. Areas in Commun., vol. 9, no. 6, pp. 785-800, 1991, doi: 10.1109/49.93089.

[21] S. M. Zafaruddin, I. Bergel, and A. Leshem, "Signal processing for gigabit-rate wireline communications: An overview of the state of the art and research challenges," IEEE Signal Process. Mag., vol. 34, no. 5, pp. 141-164, Sep. 2017, doi: 10.1109/MSP.2017.2712824.

[22] P. Golden, H. Dedieu, and K. Jacobsen, Fundamentals of DSL Technology. New York, NY, USA: Auerbach, 2006. 
[23] T. Starr, J. M. Cioffi, and P. J. Silverman, Understanding Digital Subscriber Line Technology. Englewood Cliffs, NJ, USA: Prentice Hall, 1999.

[24] H. Johnson and M. Graham, High-Speed Signal Propagation: Advanced Black Magic. Upper Saddle River, NJ, USA: Prentice Hall, 2003.

[25] G. Stockman and W. Coomans, "Fiber to the Tap: Pushing Coaxial Cable Networks to Their Limits," IEEE Commun. Mag., vol. 57, no. 8, pp. 34 39, Aug. 2019, doi: 10.1109/MCOM.2019.1800850.

[26] Assia, "G.fast: Maximum Number of Bits/Hz," ITU-T contribution, Temporary Document 11BM-028, Jun. 2011.

[27] D. H. Henao, J. Louveaux, and G. Maierbacher, "Towards a range-enhanced and spectrum-friendly g.fast," in 2016 IEEE Global Commun. Conf. (GLOBECOM), 2016, pp. 1-7, doi: 10.1109/GLOCOM.2016.7842337.

[28] Swisscom, "G.fast: Limitations on cyclic prefix length ," ITU-T contribution, COM 15 - C 2024 - E, Sep. 2012.

[29] W. Henkel, G. Taubock, P. Odling, P. O. Borjesson, and N. Petersson, "The cyclic prefix of ofdm/dmt - an analysis," in 2002 Int. Zurich Seminar on Broadband Commun. Access - Transmiss. - Netw. (Cat. No.02TH8599), 2002, pp. 22-22, doi: 10.1109/IZSBC.2002.991762.

[30] J. Verdyck, Y. Lefevre, P. Tsiaflakis, and M. Moonen, "Per-tone precoding and per-tone equalization for ofdm and dmt transmission systems: Duality, filter optimization, and resource allocation," IEEE Open J.l of Signal Process., vol. 1, pp. 257-273, 2020, doi: 10.1109/OJSP.2020.3035060.

[31] I. Almeida, A. Klautau, and C. Lu, "Capacity analysis of g.fast systems via time-domain simulations," in 2013 IEEE Int. Conf. on Commun. (ICC), 2013, pp. 4008-4013, doi: 10.1109/ICC.2013.6655186.

[32] T. Cloonan, A. Al-Banna, M. Emmendorfer, Z. Maricevic, F. O'Keeffe, and J. Ulm, "Lessons From Telco \& Wireless Providers: Extending the Life of the HFC Plant With New Technologies," in SCTE Spring Tech. Forum '15, Soc. of Cable Telecommun. Engineers, 2015.

[33] D. Acatauassu, M. Freitas, J. C. W. A. Costa, E. Medeiros, I. Almeida, and A. Cavalcante, "Coaxial Networks for 5G Fronthaul," in 2018 IEEE 23rd Int. Workshop on Comput. Aided Model. and Des. of Commun. Links and Netw. (CAMAD), Sep. 2018, pp. 1-6, doi: 10.1109/CAMAD.2018.8514967.

[34] D. Acatauassu, M. Licá, A. Ohashi, A. L. P. Fernandes, M. Freitas, J. C. W. A. Costa, E. Medeiros, I. Almeida, and A. M. Cavalcante, "An efficient fronthaul scheme based on coaxial cables for $5 \mathrm{~g}$ centralized radio access networks," IEEE Trans. on Commun., vol. 69, no. 2, pp. 1343-1357, 2021, doi: 10.1109/TCOMM.2020.3039860.

[35] IEC, "Information Technology Equipment - Radio Disturbance Characteristics Limits and Methods of Measurement," EN55022:1998 (CISPR22:1997), 1998.

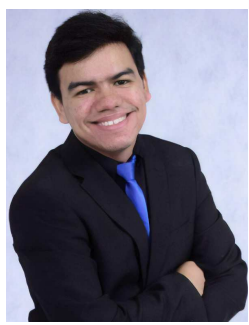

Marx Freitas received the B.S. and M.S. degrees in Electrical Engineering from the Federal University of Pará (UFPA), Belém, Brazil, in 2018 and 2019, respectively, where he is currently pursuing the Ph.D. degree with the Postgraduate Program of Electrical Engineering (PPGEE). His research interests include massive MIMO and mobile transport networks for future wireless communication systems.

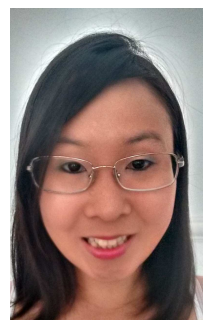

Aline Ohashi received the B.S. degree in Geophysics and the M.S. degree in Electrical Engineering from the Federal University of Pará (UFPA), Belém, Brazil, in 2015 and 2017, respectively, where she is currently pursuing the Ph.D. degree in Electrical Engineering. Her research interests include cellfree massive MIMO and non-orthogonal multiple access (NOMA).

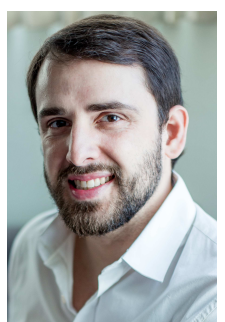

Diogo Acatauassu received the B.Sc. degree in Computer Engineering and the M.Sc. and Ph.D. degrees in Electrical Engineering from the Federal University of Pará (UFPA), Belém, Brazil, in 2006, 2009, and 2014, respectively. Since 2006, he has been with the Signal Processing Laboratory (LaPS) and Applied Electromagnetic Laboratory (LEA), UFPA, doing research on fixed access networks and small-cell transport. In the fall of 2011, he was with the Ericsson Research, Stockholm, Sweden, as a Visiting Researcher. From 2016 to 2017, he was a Professor with the Faculty of Information Systems, UFPA, Cametá, Brazil. He is currently a Professor with the Faculty of Computing, UFPA, Castanhal, Brazil. His research interests include copper-based communication and small-cell wireless communication systems, emphasizing the development of channel models for the evaluation of algorithms and system design.

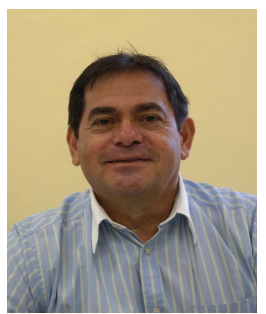

João C. W. A. Costa received the B.Sc. degree in Electrical Engineering from the Federal University of Pará (UFPA), Belém, Brazil, in 1981, the M.Sc. degree in Electrical Engineering from the Pontifical Catholic University of Rio de Janeiro, Rio de Janeiro, Brazil, in 1989, and the Ph.D. degree in Electrical Engineering from the State University of Campinas, Campinas, Brazil, in 1994. He is currently a Professor with the Institute of Technology, UFPA, and also a Researcher with the Brazilian Research Funding Agency National Council for Scientific and Technological Development, Brasília, Brazil. His current research interests include broadband systems and optical sensors.

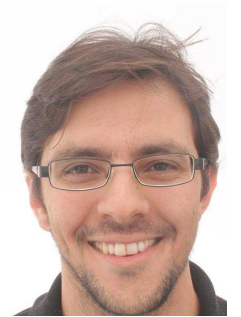

Eduardo Medeiros received the M.Sc. degree in Electrical Engineering from the Federal University of Pará, Belém, Brazil, in 2010, and the Licentiate and $\mathrm{Ph} . \mathrm{D}$. degrees from Lund University, Lund, Sweden, in 2015 and 2018, respectively. He is currently employed as a Senior Researcher with the Ericsson Research, Stockholm, Sweden. His current research interests include signal processing for broadband communications and fronthaul for 5G systems. He was a co-recipient of two best paper awards from the IEEE International Conference on Communications and three best paper awards from the IEEE Communications Society's Transmission, Access, and Optical Systems Technical Committee.

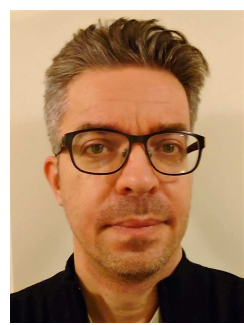

Miguel Berg received the B.S. degrees in Electrical Engineering and Computer Science from Mid Sweden University, Sweden, in 1995, followed by the Licentiate and Ph.D. degrees in wireless communication systems from the Royal Institute of Technology (KTH), Sweden, in 1999 and 2002 respectively. As a Principal Researcher at Ericsson Research, Stockholm, Sweden, he is currently leading research on efficient fronthaul and lower layer splits for $4 \mathrm{G} / 5 \mathrm{G}$ RAN. Since 2012 he has made significant contributions to research and development for the Ericsson Radio Dot System, targeting indoor small cells. After joining Ericsson in 2007, he initially worked with xDSL line testing and G.fast, as a continuation of his work as a researcher at Lund University 2006 to 2007. From 2004 to 2006, he held a research position at KTH in the area of wireless access, and from 2002 to 2003 , he worked with development of $2 \mathrm{G} / 3 \mathrm{G}$ base station antennas and tower-mounted amplifiers at Radio Components Sweden AB. He has co-authored about 35 scientific publications (journal, letters, magazine and conference), and 80 filed inventions. 


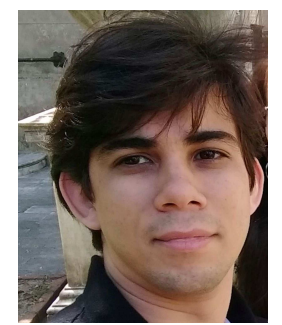

Igor Almeida received the B.Sc. degree in Computer Engineering and the M.Sc. degree in Electrical Engineering from the Federal University of Pará (UFPA), Belém, Brazil, in 2010 and 2013, respectively, where he is currently pursuing the Ph.D. degree in Electrical Engineering. He has been with the Ericsson Research since 2016, involved with topics, such as $5 \mathrm{G}$, fronthaul networking, synchronization, and wireless communications.

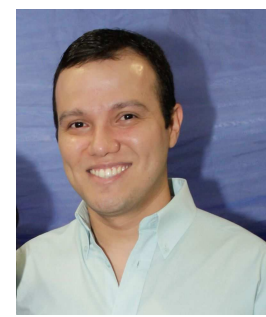

André M. Cavalcante received the Ph.D. degree in Electrical Engineering from the Federal University of Pará (UFPA), Belém, Brazil, in 2007. From 2007 to beginning of 2016, he was a Coworker with the Nokia Institute of Technology (INDT), Brazil, where he was involved in several research and development projects related to wireless communications. $\mathrm{He}$ is currently a Senior Researcher with the Ericsson Research, Brazil. His research interest includes mobile transport networks for future wireless communication systems. 